\title{
Manufacturer claims new tobacco product encourages harm reduction
}

I mperial Tobacco Canada is selling a new smokeless tobacco product in Canada, advocating its use as part of a harm reduction response to nicotine addiction.

Snus, which rhymes with "loose," is an oral tobacco packaged in small sachets that look like tea bags, although it can also be sold loose. Users place a sachet under their upper lip and absorb the nicotine through their mucous membranes. Unlike other forms of oral tobacco, users don't spit or chew it. The ground tobacco is pasteurized, which the manufacturer says reduces the amount of tobacco-specific nitrosamines it contains.

Snus use is common in Scandinavia, especially in Sweden, where it's more popular than cigarettes. According to a report by a European Commission scientific committee, a typical snus user will use a sachet for about half an hour and keep snus in his or her mouth 11 to 14 hours a day.

Imperial Tobacco is now test marketing snus in Edmonton, Alberta, where it was introduced in 2007, and in Ottawa, Ontario, under the Du Maurier label. John Clayton, Imperial Tobacco Canada vice-president of corporate affairs, says snus is a harm reduction tool because it reduces the impact of a behaviour that exposes people to risk: smoking.

"The increasing use of snus is having a net positive effect on smoking-related diseases" in Sweden, Clayton says, pointing to that country's low rate of smoking-related mortality.

Critics don't dispute that snus is less harmful than smoking, but point out that the product carries its own risks. "There is nothing you can do as dan- gerous as smoking," says Dr. Goran Boëthius, chair of Doctors Against Tobacco. "But there are effects that are serious enough," that result from snus use, including increased risk of pancreatic cancer, heart attack and stroke.

Other critics question whether the availability of snus will actually reduce

data on snus users who don't smoke.

The report attributes Sweden's low rate of smoking-related mortality to government policies from the 1950s1980s that kept smoking rates below those in Canada, where the high number of people who took up smoking then is still having an impact on mortality. While smoking rates in Canada exceeded those of Sweden in the 20th century, the report says Canada has cut down on smoking at a faster rate than Sweden, without using snus.

"Snus is not a prerequisite for success in smoking reduction, but tobacco companies make you believe that snus is the main factor that has resulted in such low smoking prevalence as we have in Sweden," says Boëthius.

But Clayton contends a lack of awareness about snus as a less harmful alternative to cigarettes has made it harder to sell the product, pointing to federal regulations in Canada that prevent the companies from advertising it. Dissolvable tobacco products, such as a film strip for the tongue, have also been introduced recently in the United States, and Reynolds American Inc. is set to launch its Camel brand snus this year.

Imperial is still assessing

smoking rates. In October 2007, Physicians for a Smoke-Free Canada released a report in response to the sale of snus in Edmonton. The Snus Experience compares tobacco use in Norway, Sweden and Canada. It found that daily smoking rates in Sweden and Norway, where snus is common, were actually higher $-25 \%$ and $35 \%$, respectively - than in Canada, where $19 \%$ of people smoke. The figures do not include the 2 Canadian test markets and has no immediate plans to expand the sale of snus, says Clayton. Meantime, Health Canada is working on regulations, expected to be in place by the fall, which will require snus packaging to carry graphic health warnings, spokesperson Jean Tessier stated in an email. Erika Gilbert, Ottawa, Ont.

DOI:10.1503/cmaj.090438 